\section{Principles of bone and joint injuries and their healing}

\author{
Tamas Nyary \\ Brigitte E Scammell
}

\begin{abstract}
This article describes the mechanisms of fracture healing (direct and indirect), general fracture management, the influence of the surgeon on the biology and biomechanical environment of bone healing, the management of articular fractures, and disorders of bone union.
\end{abstract}

Keywords Articular; bone healing; delayed union; fracture callus; non-union; open

Musculoskeletal injuries are common, accounting for 3.5 million attendances to UK emergency departments each year.

\section{Why bones fail}

A fracture is a structural failure of bone, which can occur due to a combination of extrinsic and intrinsic factors.

Extrinsic factors: the mechanism of loading will influence the likelihood of failure, due to the:

- magnitude of load

- rate of load

- direction of load.

Bone is viscoelastic, which means a time-dependent reduction in stress occurs in bone under constant strain. Therefore the rate of loading affects the extent of injury. Under higher loading speeds, as in a road traffic accident, more energy is absorbed, resulting in far greater damage to the bone and surrounding soft tissue structures (Figure 1).

Cortical bone is anisotropic, which means it has different mechanical properties when loaded in different directions. Bone may fail under applied compression, tension, shear or a combination of these forces. It is strongest in compression, weaker under tension and weakest to shear forces.

Loads applied in specific directions and rates will produce predictable patterns of failure. For example, a bone that fractures as it is pulled apart in tension will have a transverse fracture pattern, whereas one subjected to a twisting force will result in a spiral fracture pattern.

Intrinsic factors: bone is a composite made of inorganic minerals and cells surrounded by a large volume of extracellular

Tamas Nyary MD FRCS (Orth) is an Arthroplasty Fellow at Nottingham University Hospitals, UK. Conflicts of interest: none declared.

Brigitte E Scammell DM FRCS (Orth) is Professor of Orthopaedic Sciences at the Division of Orthopaedic and Accident Surgery, University of Nottingham, UK. Conflicts of interest: none declared. matrix; mainly type I collagen. It has an outer cortical layer, where the osteons are organized into compact Haversian systems and the bone is strong but brittle; and inner cancellous bone, where the Haversian systems are much less compact and separated by large areas of marrow or fat.

The relative amounts of cortical and cancellous bone determine how bones fail; for example, the calcaneum, which is mainly cancellous bone with very little cortex, often sustains a crush or compression fracture.

\section{Fracture classification}

There are a variety of classification systems available. It is important that a classification is easy to use, reproducible, predicts outcome reliably and is clinically useful for guiding management. The AO (Arbeitsgemeinschaft für Osteosynthesefragen - Association for the Study of Internal Fixation) classification is a widely used alphanumeric system of pattern recognition, used to describe individual long bone fractures. A wide variety of other classifications have been devised for describing various specific fractures.

Fractures can be broadly classified according to:

- displacement (displaced or undisplaced)

- whether the skin remains intact (closed or open)

- fracture pattern (transverse, spiral, oblique, simple, segmental or multifragmentary)

- the section of the bone involved (intra-articular, metaphysis, diaphysis)

- cause (traumatic, stress, insufficiency, pathological).

\section{Closed versus open fractures}

A closed fracture is one in which the skin is not broken. An open fracture describes an injury where a break in the skin communicates with a fracture and its haematoma. Any fracture with a wound overlying the same limb compartment must be considered open, until proven otherwise. Even the smallest of wounds is enough to allow organisms entry to a fracture site. When a bone clinically appears reasonably well aligned at the time of presentation, it may be difficult to appreciate the degree of fracture displacement that occurred during the initial injury. A bone fragment may potentially have displaced through the skin causing significant crush, stripping and devascularization of the surrounding soft tissues.

Degloving of the soft tissues is a sign of severe soft tissue injuries; it occurs superficial to the deep fascia and may result in poor viability and subsequent necrosis.

Open fractures are classified according to the Gustilo and Anderson classification (Table 1). The main factors that affect outcome are the degree of soft tissue injury and the degree of contamination of the wound.

\section{Fracture healing}

Bone differs from other tissues due to its remarkable ability to repair and heal without leaving a scar. Fracture healing is determined by mechanical and biological factors. Adult bone, unlike bone during growth, has very little ability to correct angulation or axial rotation so both must be corrected before the bone unites, or malunion will occur. 


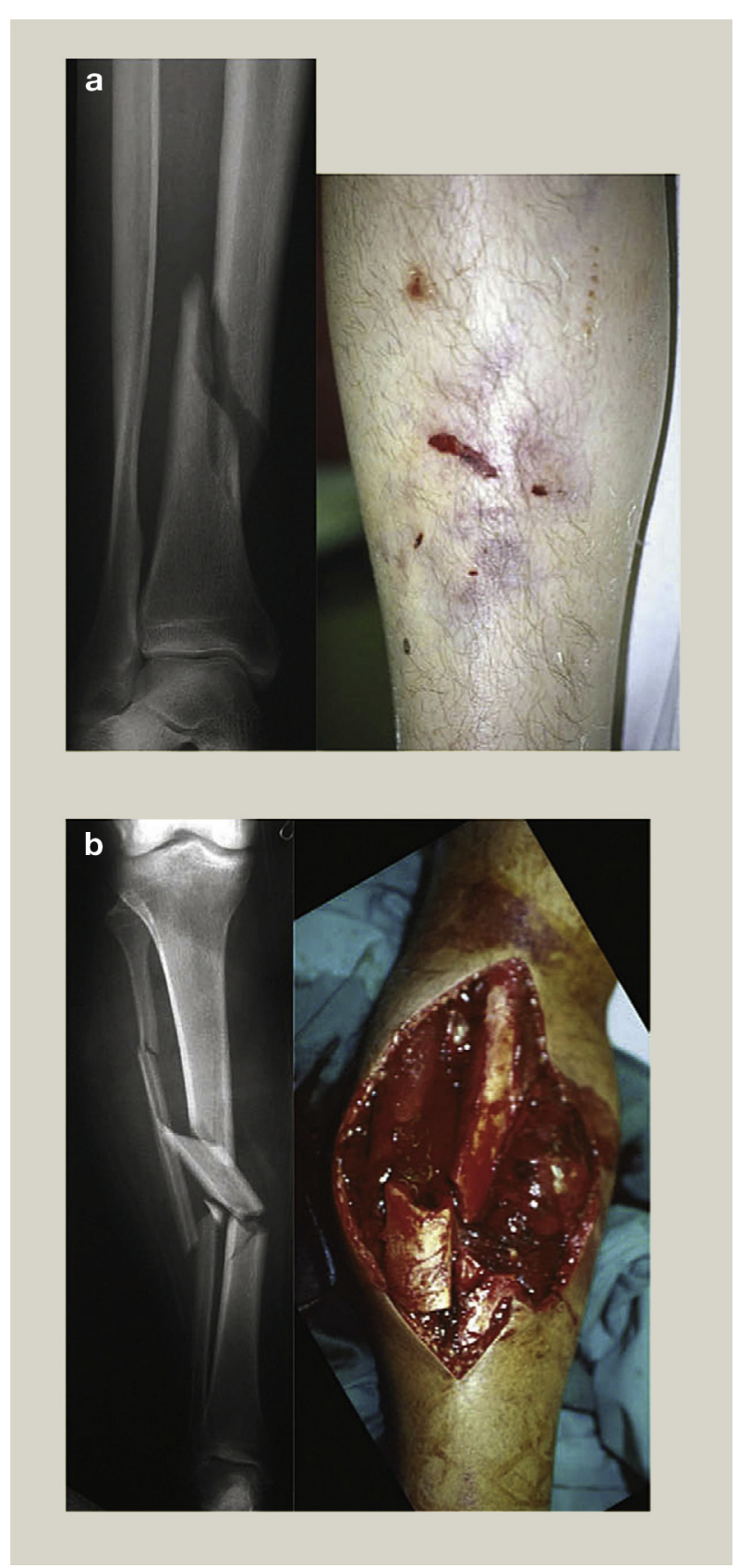

Figure 1 Effect of rate of loading to a limb. (a) At a low rate of loading, little energy is dissipated to the surrounding soft tissues. (b) At a high loading rate much surrounding soft tissue damage occurs as in this open fracture, shown post-debridement.

Healing of a fracture depends on the blood supply to the bone, the amount of force producing the fracture and the condition of the soft tissues. At the time of injury there is disruption of the Haversian systems and necrosis of osteocytes at the fracture surfaces. The extent of bone cell death depends on the degree of fracture comminution and displacement, disruption of the local blood supply and the extent of periosteal stripping (which affects the cortical blood supply and removes the cambial layer of periosteal stem cells from the surface of the bone). In general, the greater the damage to bone and surrounding soft tissues, the slower the bone is to heal.
The pattern of bone healing can be modified by the mechanical environment of the fracture and this can in turn be manipulated by surgical intervention. The purpose of fracture stabilization is to maximize the biology of fracture healing to aid early union and restore function while minimizing complications.

Direct (primary) healing: direct or primary healing can be achieved through surgery; with anatomical reduction of the fracture and application of rigid internal fixation to generate absolute stability (no interfragmentary motion under physiological load). This generates a very low interfragmentary strain.

With a fracture gap of less than $200 \mu \mathrm{m}$ and absolute stability, osteoclasts can tunnel across the fracture line, establishing a 'cutting cone' between the bone ends (Figure 2). Osteoblasts follow, laying down bone matrix and re-establishing continuity between the Haversian systems. Revascularization occurs, with internal bone remodelling, and bone forms directly with no callus formation.

Direct fracture healing with the formation of new cortical bone occurs slowly, and is essentially the same biological process that occurs in normal bone turnover and remodelling.

Indirect (secondary) healing: the majority of fractures heal by indirect healing. This occurs in an environment of relative stability (where some controlled interfragmentary motion still exists under functional loading). The stages of repair last several weeks, and are described in three sequential phases (Figure 3), as follows.

\section{- Reactive phase}

- Inflammation and haematoma formation. Bone has an excellent blood supply; the medullary cavity and inner two-thirds of the cortex are supplied centrifugally by endosteal arteries within the bone, whereas the outer third of the cortex is supplied by the periosteal arteries. Bleeding from the bone and surrounding soft tissues results in haematoma formation with a clot of insoluble fibrin at the fracture site.

- The clot provides a framework of fibrin fibres for the influx of various migrating cells (e.g. neutrophils, lymphocytes, monocytes, macrophages, mast cells, platelets). These cells release various cytokines, including transforming growth factor- $\beta$, platelet-derived growth factor, fibroblast growth factor, and interleukins 1 and 6 . Histamine is released from mast cells and platelets. This environment encourages an increased capillary permeability, chemotaxis and small vessel dilatation.

- Granulation tissue formation. The initial fracture has significant interfragmentary motion, which generates a significant interfragmentary strain. (Strain is defined as the extent of deformation relative to its initial condition, i.e. a relative measure of displacement of particles as a force is applied.) Granulation tissue forms, as it tolerates conditions of high strain (up to $100 \%$ ), whereas bone cannot form at this stage, as osteoblasts only tolerate very low strain $(<1 \%)$. The granulation tissue matures, reducing strain across the fracture site. Osteoclasts begin to resorb the dead bone ends and phagocytes remove other necrotic tissue. 


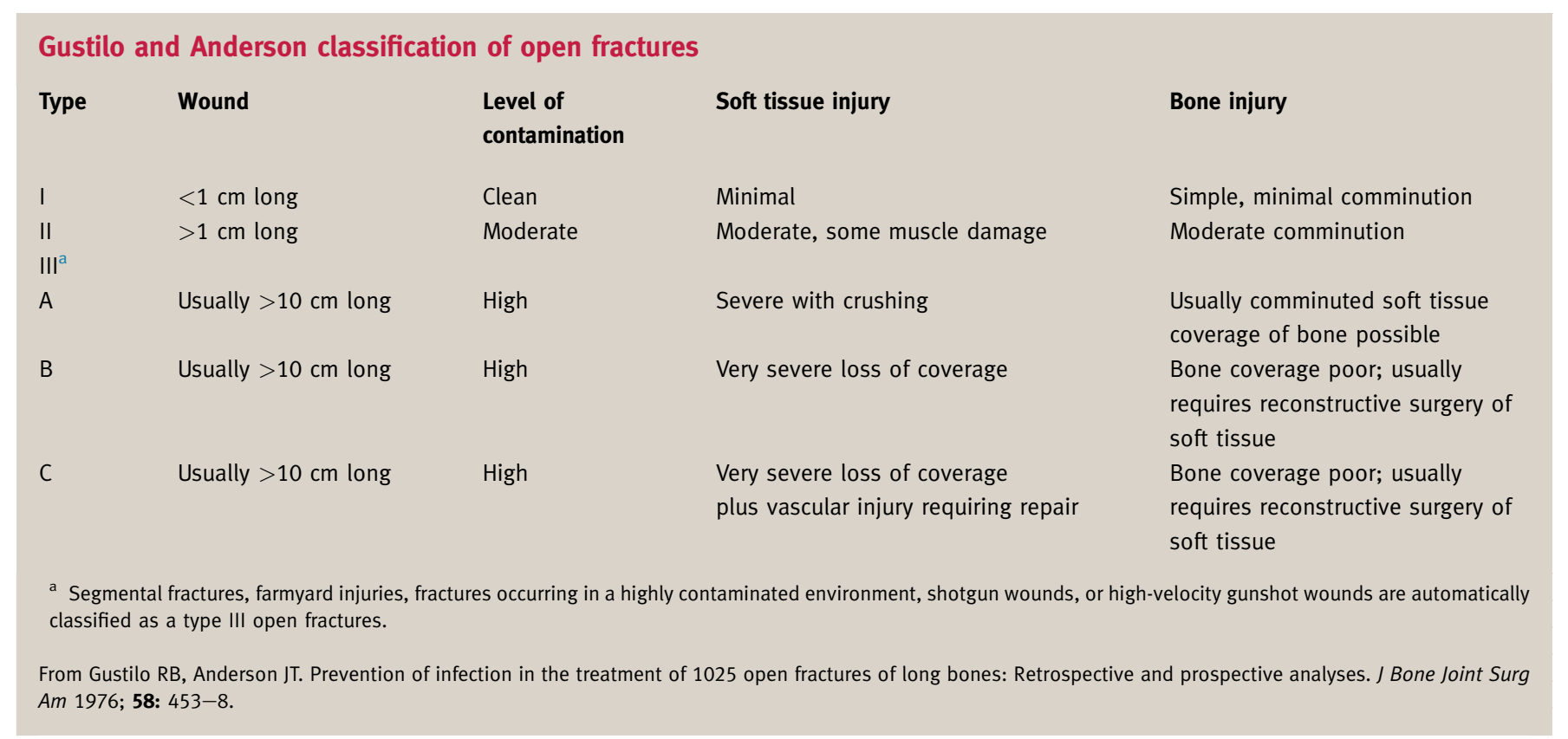

Table 1

- Reparative phase

- Callus formation. Cartilaginous callus bridges the fracture site within 2 weeks, once the interfragmentary strain falls below $10 \%$. If the bone ends are not touching bridging callus will form. Type II collagen is produced early in fracture healing within the cartilage matrix and is followed by type I collagen expression as new bone.

- Cyclical micromotion stimulates the growth of cartilage and then bone. The optimal size of these movements is about $1 \mathrm{~mm}$. As the callus grows in size, it becomes stiffer, thus facilitating osteogenesis.

o Generally, the amount of callus formed is inversely proportional to the stability of the fracture. However, extremely unstable fractures will not unite, as the strain remains too great, ossification fails and only fibrous union can occur.

-Woven bone. Endochondral ossification is the process of mineralizing cartilaginous callus and is apparent on early radiographs, This 'woven bone' forms once the strain falls below $1 \%$. The chondrocytes in the cartilaginous matrix become hypertrophic, calcify and die, allowing angiogenesis to occur. Osteoblasts lay down woven bone on this collagen framework left by the chondrocytes. Cyclical micromotion stimulates the growth of the callus.

- Remodelling phase

- Trabecular bone. Once the fracture has been satisfactorily bridged by callus, the newly formed bone is remodelled. Any excess callus is removed and the woven 'osteoid' bone is remodelled into trabecular 'lamellar' bone. The medullary canal and bone shape are subsequently restored. Remodelling continues long after the fracture has clinically healed (up to 7 years).

Additional bone is gradually laid down in areas of mechanical stress and removed from areas where there is too little. This remodelling, which is really just an extension of normal bone turnover, is known as Wolff's law.

Children: a child's bone is significantly different to that of adults. They have physeal plates located at the ends of the long bones, responsible for continued longitudinal growth. Compared to adult periosteum, a child's periosteum is thicker and stronger; this dense fibrous membrane can impart some stability to the fracture, as well as preserve the vascular supply necessary for rapid repair.

A child's bone is more elastic and plastic, allowing it to deform considerably before breaking which may generate different fracture patterns to those of an adult.

Remodelling is usually very effective. Even completely displaced fractures may heal and remodel without trace. The younger the child, the greater the potential for remodelling, and the physis of a bent bone will grow eccentrically to help restore alignment. Growth in bone length and width will conceal deformity. There is some ability to correct angulation but this decreases in adolescents. Axial malrotation can never be accepted, as it will not remodel.

\section{Patient management}

This requires an assessment of the whole patient, then focus on the injured limb, followed by definitive fracture management.

\section{- Initial assessment and management}

The Advanced Trauma Life Support (ATLS) protocol of airway, breathing and circulation must be applied to all trauma patients. The history may determine the direction and magnitude of the force applied to the broken bone.

\section{- Assessment of the broken bone}

Clinical examination must be systematic and repeated, to identify established and evolving limb-threatening conditions. This must include assessment of the soft tissues and the distal 


\section{Primary bone healing}

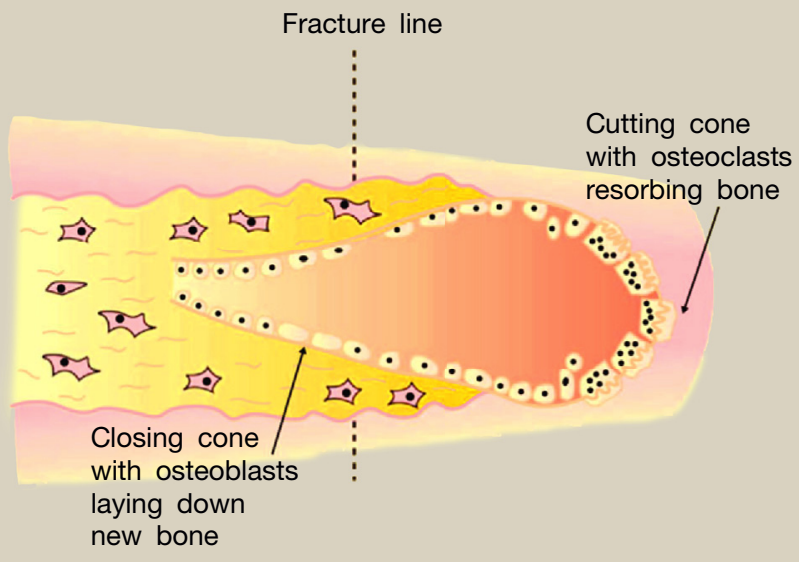

Figure 2 Direct bone healing. A cutting cone tunnelling from left to right. The cutter head is at the right with multinucleated osteoclasts to resorb the dead bone. The tail, with its conical surface, is lined with osteoblasts (as seen on the left) laying down new bone. This is a slow process which is also seen in normal turnover of bone. neurovascular status of the limb. Radiological assessment should include the whole of the fractured bone, typically for long bone fractures this includes the joint above and below.

The condition of the soft tissue surrounding the fracture dictates management, even in closed fractures. Operating through bruised and highly swollen tissues should be avoided, as the wound may be impossible to close or subsequently break down (Figure 4).

\section{- Management of open fractures}

Haemorrhage is controlled by direct pressure or, as a last resort, by a tourniquet. Broad-spectrum antibiotics should be administered as soon as possible after the injury, and certainly within 3 hours. Tetanus vaccination status should be checked and booster injection should be considered. Any gross contamination should be removed, the wound photographed, adequately splinted and covered with sterile dressings until formal debridement can be performed in an operating theatre. No provisional cleaning of the wound should be performed before surgery.

Complex open fractures require a combined orthopaedic and plastic surgical opinion, and may need immediate referral to a regional specialist centre. The primary surgical procedure should ideally be performed at the same centre that all further surgery is to occur, to ensure appropriate planning and continuity.

\section{Secondary fracture healing: bridging of a fracture by external callus}

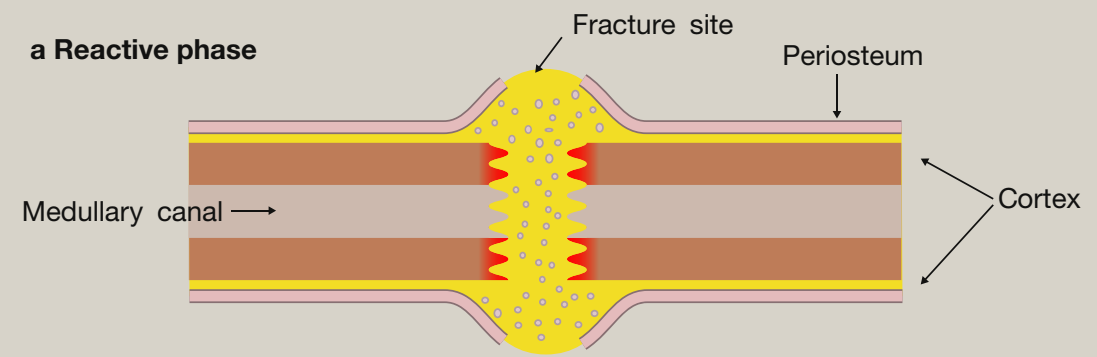

Haematoma forms at the fracture site and the periosteum is torn. The bone ends die and are resorbed by osteoclasts

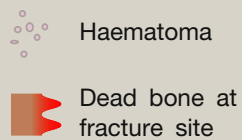

b Reparative phase

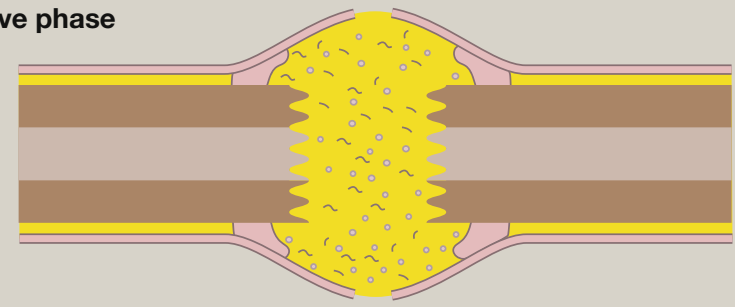

c Remodelling phase

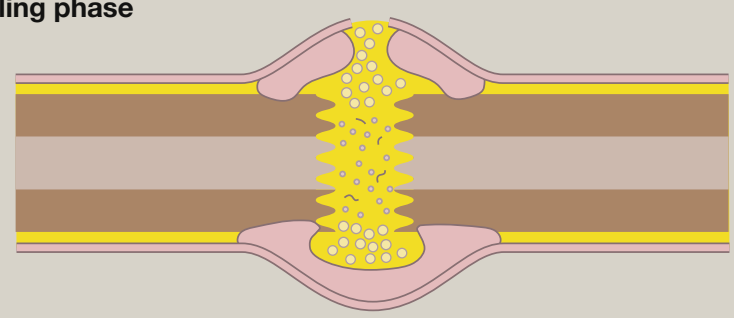

Granulation tissue replaces the haematoma and woven bone or hard callus starts to form the abutments of the bridge from the cambium layer of the periosteum by intramembranous ossification

$\therefore$ Granulation tissue

New woven bone/ external hard callus

The fracture gap is bridged by soft callus or cartilage. This is replaced with bone by the process of endochondral ossification. The gap is also bridged by hard external callus arching over the soft cartilaginous callus as shown in the lower half of the diagram. Internal or medullary callus forms more slowly and finally cortical continuity is restored

\section{Cartilage/soft callus}

Figure 3 


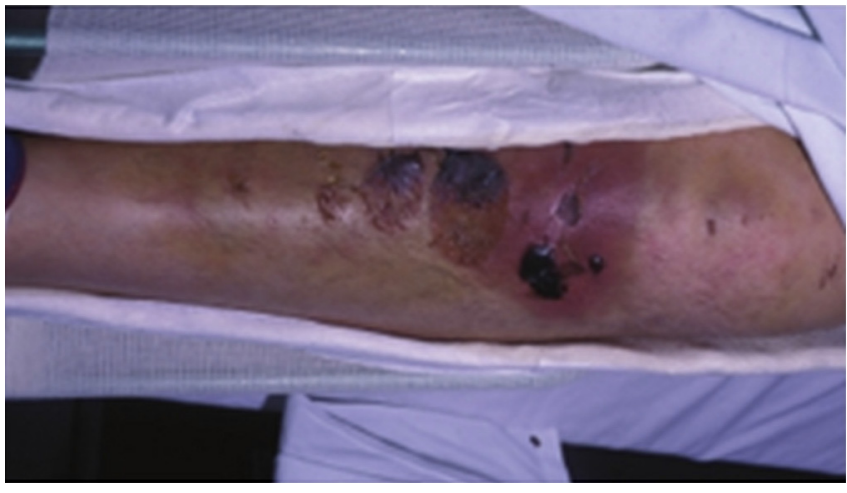

Figure 4 Closed fracture with severe bruising. This fracture of the tibial plateau occurred 4 days ago, but the degree of skin blistering and bruising indicate that it is too early to consider open reduction.

The only reasons for immediate surgical exploration are:

- removal of gross contaminants

- compartment syndrome

- a devascularized limb

- a multiply injured patient going to theatre.

The principles of a debridement include wound extension to determine the extent of the injury, removal of all devitalized tissues (except neurovascular bundles), including bone, followed by adequate irrigation with warmed fluids to reduce the bacterial load (often at least 6 litres is required for a long bone injury). High-pressure lavage is not recommended as it may add to the soft tissue damage already present and seed bacteria into the bone medulla. Patients with highly contaminated wounds and severely damaged soft tissues should return to theatre every 48 hours until the wound is clean and only healthy tissues remain.

\section{- Definitive fracture management:}

The principles of definitive fracture management are reduction, stable fixation (external or internal), preservation of blood supply, and early mobilization (rehabilitation). There are different methods for treating the same fracture, but regardless of the means used, basic principles need to be followed in order to avoid poor outcomes. Reduction can be achieved by closed (traction and manipulation) or open methods (surgery). Stabilization is achieved by relative or absolute stability, using nonoperative, internal or external fixation techniques.

\section{- Relative stability:}

Plaster casts: prevent angulation and malrotation. Indirect fracture healing takes place with callus visible on radiographs. However, only transverse fractures have axial stability in a cast, and oblique fractures may displace and shorten (Figure 5a).

Traction: is an effective and safe way of maintaining reduction in some clinical situations; however, improvements in fracture fixation methods in conjunction with holistic care of injured patients has seen this method of fracture treatment decrease. Early mobilization of patients following surgery decreases the complications associated with the prolonged period of bed rest required. Healing is by abundant callus formation.

External fixation: is particularly useful if there is a surrounding soft tissue injury. All external fixators allow movement at the
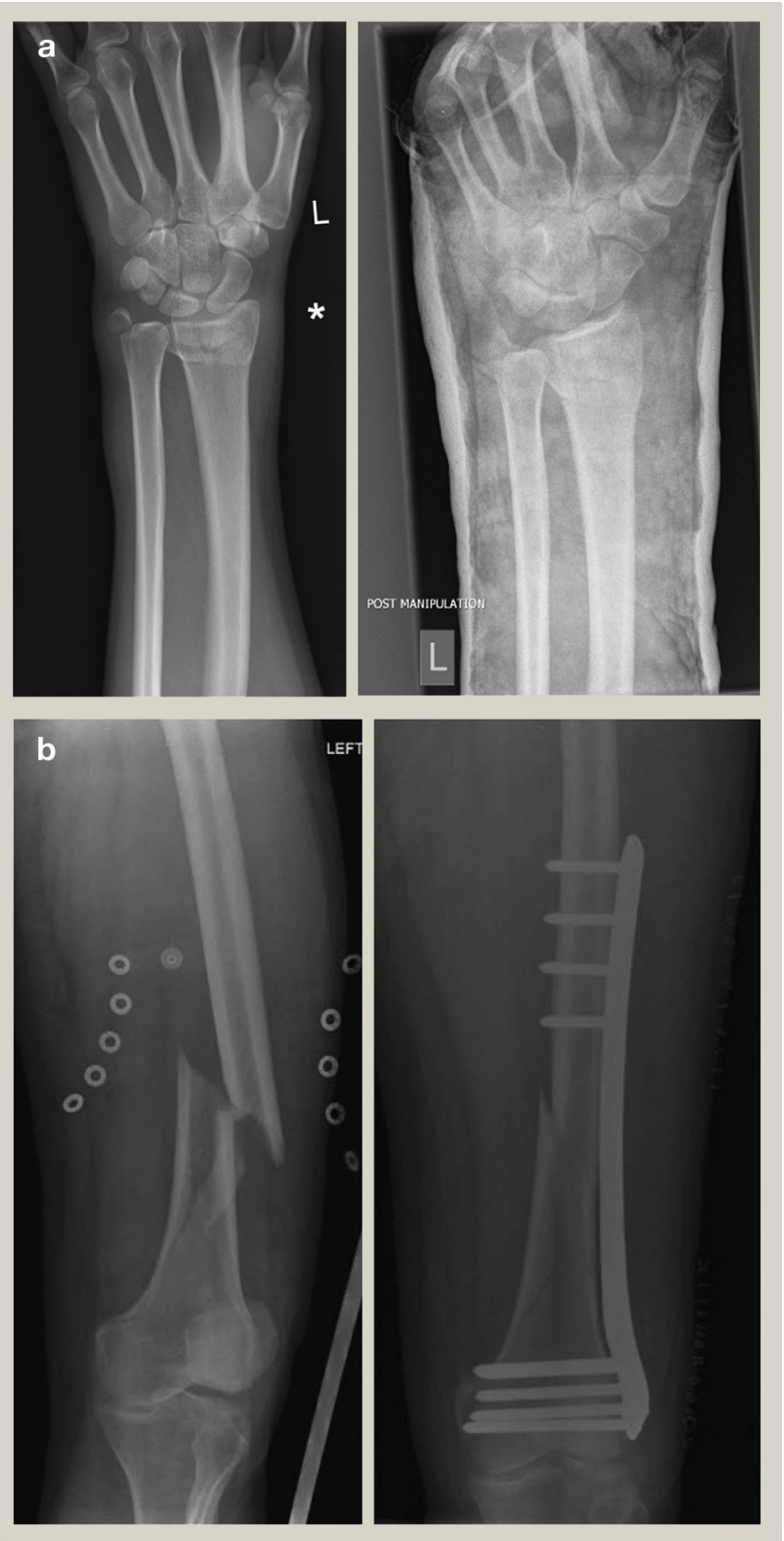

Figure 5 Relative stability fixation techniques. (a) Multifragmentary fracture of the distal radius with associated fracture of the ulna styloid stabilized by plaster cast. (b) Fracture of the distal third of the shaft of the femur stabilized with a bridging plate. Both of these techniques will result in indirect healing of the fracture with external callus formation.

fracture site, so promote healing with callus formation. Circular frames are particularly useful if the fracture is very close to a joint and associated soft tissue injuries preclude internal fixation. They provide stability in three planes and allow axial micromovement to encourage callus formation.

Internal fixation: generally can be used to permit some controlled motion at the fracture site and encourages callus formation. Indications for internal fixation include displaced and intra-articular fractures, fracture instability (axial, rotational, angular) and associated neurovascular injury. 


\section{Examples are}

Wire fixation - wires are strong enough to support fracture segments but do not resist forces acting perpendicular to the fracture line.

\section{Plate and screw fixation}

- Buttress plates - resist axial load by applying a perpendicular force to the potential deformity.

- Tension plates - on the tensile surface of the bone resist tensile forces when the bone is loaded; the tension plate configuration also allows dynamic compression on the far bone cortex if the fracture is anatomically reduced.

- Bridging plates - secure the two main fragments, leaving the fracture zone undisturbed (so called 'biological plating') (Figure 5b).

- Locking plates - the screw engages and 'locks' into the plate with a mechanism which secures a fixed angle between the two. The plate does not have to contact the bone avoiding avascular damage to periosteal structures.

- Intramedullary nails prevent angulation and provide axial stability. Locking screws add rotational stability. Some residual motion remains at the fracture site, stimulating bridging callus formation (Figure 6).

\section{- Absolute stability:}

Internal fixation can be used to achieve anatomical reduction and fixation with absolute stability. Interfragmentary compression prevents motion between the fracture surfaces under functional load; there is a very low strain across the fracture and direct bone healing occurs without formation of external callus. This is a slow process that relies on internal remodelling of the bone. Interfragmentary compression can be achieved with a lag screw across the fracture or a dynamic compression plate that causes compression as the screws are tightened (Figure 7).

The technique of anatomical reduction with absolute stability is required in particular situations:

- displaced intra-articular fractures - to reconstruct the joint surface

- forearm fractures - pronation and supination will be reduced unless anatomical reduction is achieved

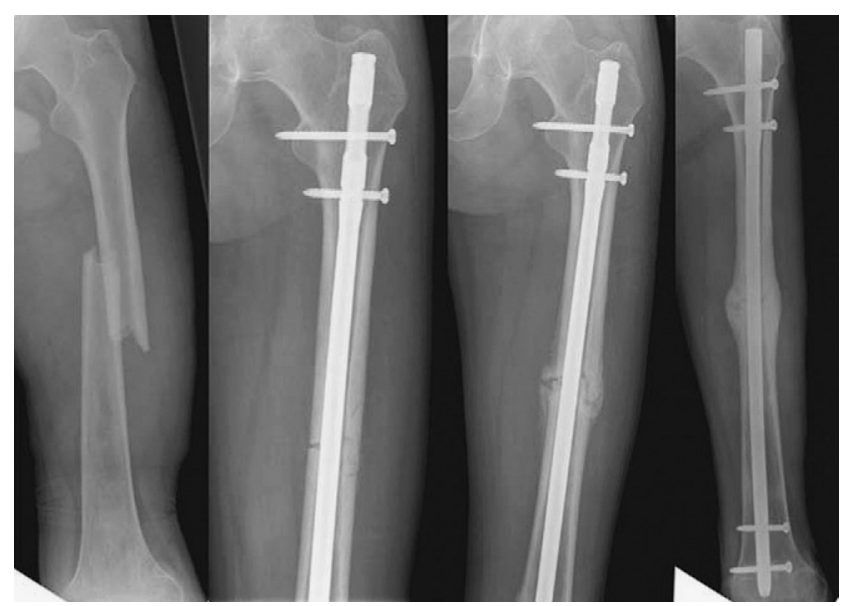

Figure 6 Indirect bone healing. A mid-shaft femoral fracture treated with a locked intramedullary nail. Union progresses by indirect healing with external callus (from left to right). The third image shows both 'hard' external callus which is mineralized and therefore easily visible, with central 'soft' callus that is cartilaginous.
- in the management of non-union.

\section{Fractures involving a joint}

Intra-articular fractures may result in stiffness, deformity, pain, and post-traumatic arthritis. In order to restore function and minimize deformity and deterioration it is necessary to secure an anatomical reduction of the articular surface, restore joint stability and axial alignment, and begin early joint motion.

An intra-articular fracture is a complex injury, of which the fracture forms just one part. From the perspective of immediate or early complications, failure of the soft tissue component possess the greatest risk of poor outcome.

\section{- Assessment}

A thorough clinical examination is essential to assess neurological deficit, vascular injury, compartment syndrome, and tenderness over the course of the surrounding structures; this may be the only clue of ligamentous disruption. Radiographs are necessary for defining the extent of a fracture, although articular detail can be obscured by overlap of the fragments. A CT scan is frequently required to assess the articular components. An MRI scan should be considered in order to assess the associated soft tissue structures in more detail.

\section{- Timing}

As described below, early surgery through soft tissues traumatized by a high-energy injury is highly associated with a high incidence of wound healing problems and infection. The only intra-articular injuries which demand immediate surgical intervention are:

- open fractures

- fracture and/or dislocation with neurological deficit or skin compromise, due to the displaced bone.

- fracture and/or dislocation with neurological deficit or skin compromise, due to the displaced bone.

\section{- Approach and technique}

Once the characteristics of the fracture have been defined by clinical and radiological investigation, it is necessary to make a careful preoperative plan for surgery. This includes a plan of the surgical approach as well as a detailed plan of the internal fixation and any bone grafting required.

The surgical reconstruction of a joint requires anatomical reduction of the articular surface, with minimal soft tissue disturbance. This often requires depressed portions to be elevated from their impacted position in the metaphysis. Once elevated, the fragments must be held provisionally in their reduced positions using wires, bone grafting of the metaphyseal cancellous defect may be required before application of the definitive internal fixation to the bone.

Following surgery for intra-articular injuries, the joint should commence immediate passive mobilization; a continuous passive motion (CPM) machine can be utilized. However the merits of mobilization must be balanced against the degree of stability obtained at the time of surgery.

\section{Factors affecting healing}

\section{- General factors}

- Age. Children's bones unite more rapidly; the speed decreases as skeletal maturity approaches. Children's 


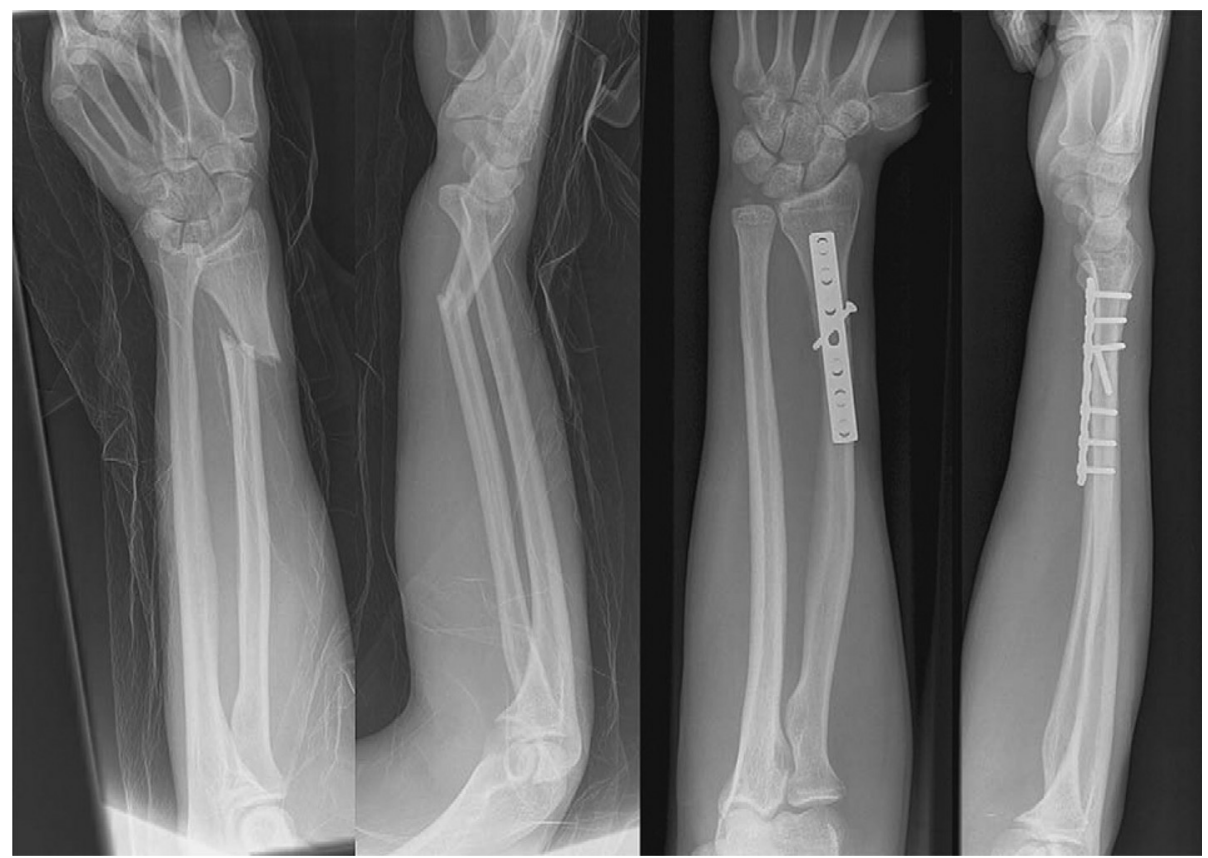

Figure 7 A Galeazzi fracture. Absolute stability is provided by the lag screw across the fracture protected by a plate. The fracture united without external callus.

bones also have great capacity for remodelling (except for axial rotation).

- Nutrition and drug therapy. Poor nutrition and general health reduce rates of healing. Smoking, and use of corticosteroids and non-steroidal anti-inflammatory drugs impair the inflammatory response and delay bone healing.

- Bone pathology. Pre-existing bone disease such as malignancy inhibits bone healing and screws in osteoporotic bone often fail.

- Bone type. Cancellous bone tends to heal faster than cortical bone. This is due to a large area of bony contact (e.g. in the metaphysis) and the greater number of active bone cells present.

\section{- Local factors}

- Mobility at fracture site. Excess mobility at the fracture site will interfere with vascularization of the fracture haematoma, cause high strain and disrupt the bridging callus, thus interfering with union.

- Separation of the bone ends. Bony union may be delayed or prevented if the bone ends are separated by interposed soft tissue, or held apart with the fixation device or traction.

- Disturbance of blood supply. A fundamental factor affecting healing is the blood flow to the bone. Fractures compromising blood flow to the fracture site or one of the fragments at the fracture are slow to unite if they unite at all. Examples include intracapsular fractures of the neck of femur and scaphoid fractures where the blood supply to the bone is via an end artery. Highenergy axial compressive injuries dissipate much of the forces through their soft tissue envelope. This results in comminution, loss of soft tissue attachments, periosteal stripping and delayed healing. In these closed injuries the biology of the compromised fracture site must be respected, and temporary stabilization is best achieved with plaster or external fixation devices. Definitive surgery should be delayed until the soft tissue envelope has recovered, which can take as long as $2-3$ weeks.

- Bone properties. There is a variation in the speed at which bones heal in the same individual. Fractures of the upper limb generally heal more quickly than fractures in the lower limb. Clavicle fractures heal remarkably well; whereas tibial shaft fractures heal slowly.

- Fracture type. Displaced and comminuted fractures frequently result in delayed healing. The avascular fragments of splintered bone require resorption, a more extensive inflammatory and callus phase, and more time to remodel. Transverse fractures take longer to heal than spiral fractures because they usually have more displacement of the periosteum and a smaller surface area of contact.

- Infection. The presence of infection in a fracture will delay or prevent healing. There is a prolonged inflammatory phase and cellular activity is directed towards fighting the infection rather than bone healing. Additionally, in the presence of metalwork, bacteria readily produce biofilms (a protective mucopolysaccharide layer), which render normal antibiotic dosage regimens ineffective, toxic concentrations of antimicrobials would be required to inhibit bacterial growth.

- Biomechanical environment. There is an optimal balance between stability and micromotion to encourage callus formation. Small, cyclical movement of about 1 $\mathrm{mm}$ increase the rate of healing by $25 \%$ and are a feature of many external fixation devices.

- Ultrasound. Low-intensity pulsed ultrasound accelerates fracture healing, and improves mechanical strength by 


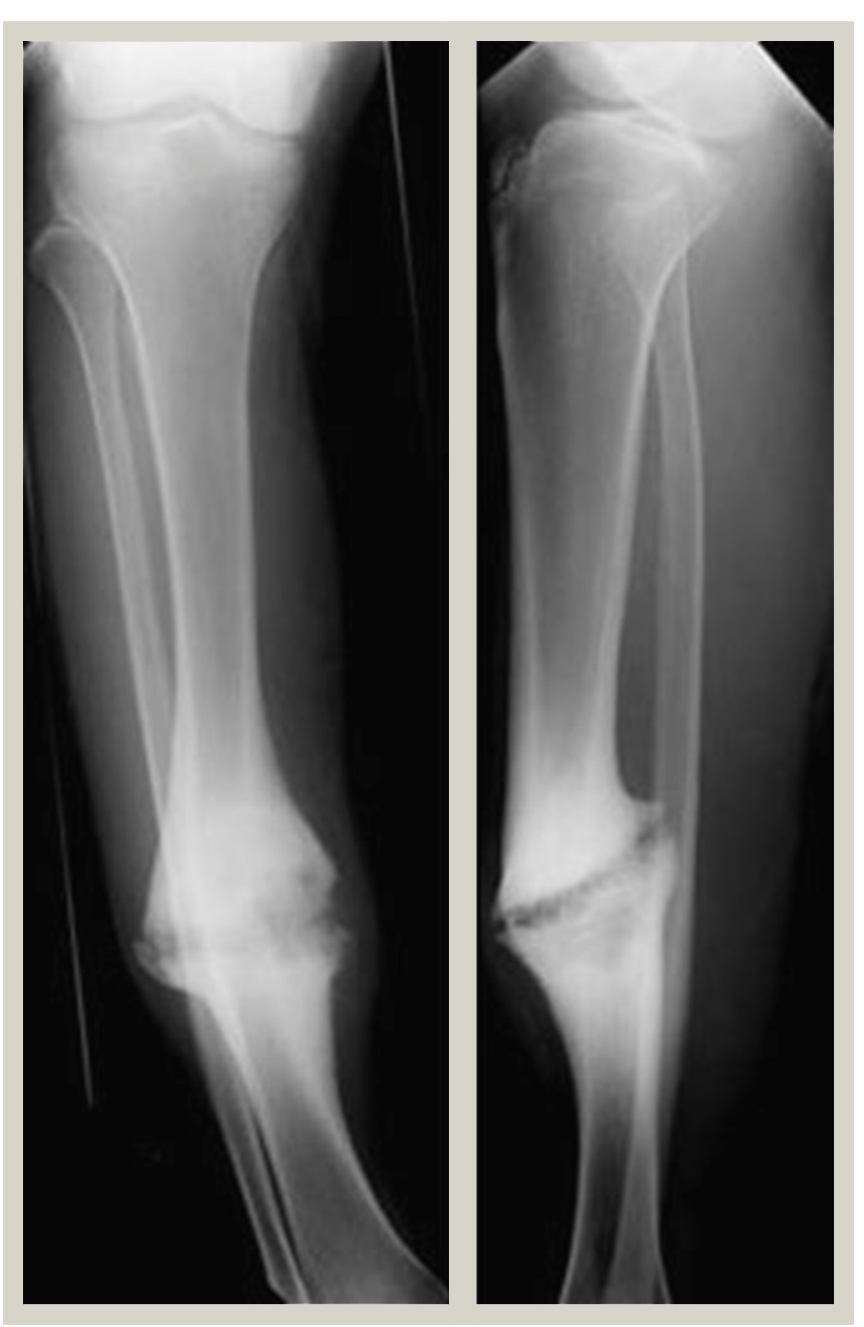

Figure 8 Hypertropic non-union of a tibia fracture. Sclerotic bone ends have a typical radiographic appearance of an 'elephant's foot'.

increasing the stiffness and torque of fracture callus. Its use is controversial.

- Electromagnetic environment. Direct current helps stimulate an inflammatory-type response and pulsed electromagnetic fields initiate calcification of fibrocartilage. Again, therapeutic use is controversial.

- High-dose irradiation. Associated with a decrease in cellularity, long-term changes within the Haversian system and an increased risk of non-union.

\section{Disorders of bone union}

\section{Delayed union}

Delayed union happens when healing fails to occur within the expected time for the fracture, despite optimal treatment. The fracture proceeds through the normal stages of healing clinically and radiologically but at a slower rate. This can be due to intrinsic factors (tibial diaphyseal fractures are often slow to unite), a reduced blood supply or infection at the fracture site. Absolute stability in the presence of a fracture gap greater than 1 $\mathrm{mm}$ will delay union. The rigid fixation will inhibit healing by callus formation and the gap will delay or even inhibit direct bone healing.
Eventually, the healing process ceases to be active and if by 6 months there is no bridging callus, the case has progressed to non-union.

\section{Non-union}

Non-union occurs if there is wide separation of the bone ends, soft tissue interposition, poor blood supply, infection or an adverse biomechanical environment. There are two types of non-union:

- Hypertrophic non-union: Inadequate stability of the fracture leads to hypertrophic non-union. On radiographs, the viable bone ends appear sclerotic and flared owing to excess callus formation; this gives the pathognomonic radiological appearance of an 'elephant's foot' (Figure 8). Histologically the gap between bone ends is filled by cartilage and fibrous tissue. The blood supply at the bone ends is good and immobilizing the fracture (e.g. with an intramedullary nail) usually results in union.

- Atrophic non-union: There is no attempt at healing, the bone ends are resorbed and rounded; this represents a failure of bone biology. The gap between the bone ends is filled by fibrous tissue. Rigid fixation with interfragmentary compression and elimination of the fracture gap is required, and supplementary bone grafting may be required. Recombinant human bone morphogenetic proteins are now commercially available and are licensed for use in difficult non-union surgery.

\section{Summary}

When managing musculoskeletal injuries, it is important for the surgeon to have a broad understanding of:

- the appropriate description and classification of fractures

- the mechanisms of direct and indirect bone healing, and the stages of indirect healing

- how a paediatric bone responds differently to injury

- open fractures and their initial management requirements

- articular fractures and their surgical management

- definitive fracture management options with relative or absolute stability.

\section{FURTHER READING}

Hahn DM. Current principles of treatment in the clinical practice of articular fractures. Clin Orthop Relat Res 2004; 423: 27-32.

McRae R, Esser M, eds. Practical fracture management. 4th edn. Edinburgh: Churchill Livingstone, 2002.

Rüedi TP, Buckley RE, Moran CG, eds. Practical fracture management. 2nd edn. New York: Thieme, 2007.

Standards for the management of open fractures of the lower limb. 1st edn. London: BAPRAS and BOA, September 2009 . http://www. bapras.org.uk/downloaddoc.asp?id=141.

Standing S, ed. Gray's anatomy, the anatomical basis of clinical practice. 40th edn. Edinburgh: Churchill Livingstone, 2008.

\section{Acknowledgements}

Thanks to Professor Christopher Moran, Mr Nitin Badhe and Mr Tom Kurien for their assistance with the images used in this article. 